\title{
Reply to Professor Lykken
}

\author{
Ronald S. Wilson \\ University of Louisville, Louisville, Kentucky
}

Professor Lykken's letter of clarification is an excellent example of why my method was recommended as the simplest and most direct. In his third paragraph, Lykken gives a brief summary of how the probabilities and odds are figured and transformed back and forth to arrive ultimately at the desired figure.

The logical problems in this approach have been examined in detail in Wilson [2:113-115], and space does not permit a full review here. The reader is referred to the previous article for a complete analysis. Perhaps it is sufficient to note that the complex derivation of the probability of $\mathrm{DZ}$ concordance for each phenotype rests on the ratio of the two-zygote concordance value to the proportion of two-zygote pairs in which the random prospectus displays the reference phenotype. It is not a calculation that provides a basic sense of understanding about the essential probabilities involved.

Since the diagnostic issue arises only for concordant pairs, the crucial diagnostic question may be phrased as follows: Given the specific array of bloodgroup phenotypes that the twins display, how might this array have been obtained by a DZ pair, and how might it have been obtained by an $\mathrm{MZ}$ pair? The phrasing of the question is crucial, since it is the probability for the entire phenotype array that determines the ultimate likelihood of the pair being $\mathrm{MZ}$.

In terms of computation, two probabilities are computed for each concordant phenotype-the first on the hypothesis of $\mathrm{DZ}$ origin, and the second on the hypothesis of $\mathrm{MZ}$ origin. The resultant values are then cumulatively multiplied across phenotypes to yield two composite $p$ values for the entire array. These two composite $p$ values furnish the essential figures needed for the final zygosity equation (Step 2).

Since the necessary probabilities for all phenotypes in the eight major bloodgroups are tabled in Wilson [2:Table 3], the investigator needs only to select the appropriate values from the table to perform the computations. Lykken's remark about my method being cumbersome is gratuitous - no investigator needs to labor through it for the bloodtyping data typically available. One reason for giving the method in such extensive detail was to show the precise steps leading from initial gene frequencies to the final estimate of two-zygote concordance for a given phenotype. My belief is that it gives a much clearer understanding of exactly how the probabilities are obtained than any other method.

There are computational shortcuts that can considerably reduce the amount of calculation needed for any two-allele system. Indeed, the layout in Appendix 1 [2] provides 
a ready model for substituting other gene frequencies and computing the necessary probabilities. If other serum proteins or enzymes are typed and the pair is concordant, E can either employ Lykken's method and tabled values, or else be assured that the likelihood of $\mathrm{MZ}$ origin is even greater than that indicated by the red-cell typing alone.

As to the issue of variation in gene frequencies and its effect upon zygosity estimates, Table 5 of Wilson compared three different white samples and found a high degree of similarity between all three sets of values. The minor variations in gene frequencies among these white populations (in England, Kentucky, and Minnesota) had only a negligible effect upon the concordance values for DZ twins, and thus the tabled entries in Wilson may be used with confidence for white twins.

For other ethnic groups such as blacks, the gene frequencies do differ for certain phenotypes, and there are as yet no tabled concordance values specifically for such groups. If an exact $p$ value is needed for a particular pair, then $\mathrm{E}$ either employs my method or the Lykken/Sutton equations to obtain the needed figure.

In most cases, however, $\mathrm{E}$ is simply using the concordance/discordance split on bloodtyping to subdivide the twin sample into $\mathrm{MZ}$ and $\mathrm{DZ}$ pairs. A gene frequency change is of minor consequence to this issue unless $\mathrm{E}$ has determined in advance to classify certain concordant pairs as $\mathrm{DZ}$ rather than $\mathrm{MZ}$ if their $p$ value does not reach a specified level. In that case, $\mathrm{E}$ would want to identify the affected phenotypes and recompute the needed values, plus make a change in the expected $\mathrm{MZ} / \mathrm{DZ}$ ratio in the population, since blacks have a higher proportion of $\mathrm{DZ}$ twins.

To return to the original issue - all methods, if correctly employed, will ultimately yield the same probability of being $\mathrm{DZ}$ or $\mathrm{MZ}$ for a given concordant pair. There are significant differences in logic and application, however, especially in deriving the computational method for the individual case. Readers are invited to consult the original papers to see which derivation is more direct and comprehensible $[1,2]$.

\section{REFERENCES}

1. Lykken, DT (1978): The diagnosis of zygosity in twins. Behav Genet 8:437-473.

2. Wilson, RS (1980): Bloodtyping and twin zygosity: Reassessment and extension. Acta Genet Med Gemellol 29:103-120. 